EPJ Web of Conferences 41, 02029 (2013)

DOI: $10.1051 /$ epjconf/20134102029

(C) Owned by the authors, published by EDP Sciences, 2013

\title{
Probing chirality with a femtosecond reaction microscope
}

\author{
N. Bhargava Ram, C. S. Lehmann and M. H. M. Janssen
}

LaserLaB, VU University Amsterdam, 1081HV Amsterdam, the Netherlands

\begin{abstract}
Detection of molecular chirality with high sensitivity and selectivity is important for many analytical and practical applications. Photoionization has emerged as a very sensitive probe of chirality in molecules. We show here that a table top setup with a femtosecond laser and a single imaging detector for both photoelectrons and photoions enables detection of chirality up to 3 orders of magnitude better than the existing conventional absorption based techniques.
\end{abstract}

\section{Introduction}

Chiral molecules are a special class of molecules that exist in two forms (or enantiomers) - the left and right handed form - with the same constituent atoms or groups but are mirror images of each other and have different properties arising solely due to their difference in handedness. Chirality is one of the most fundamental and ubiquitous concepts of life. Important biological molecules such as amino acids and sugar bases in living systems and many pharmaceutical drugs are chiral. Further, it is seen that only one form - either left or right - prevails or is useful in most cases. Medicinal value in most pharmaceutical compounds is found only in one enantiomer, while the other form could be harmless but sometimes cause serious side-effects. Many procedures and applications need separation of the two chiral forms with high selectivity and sensitivity.

Optical detection of chirality and its molecular structure is conventionally performed by optical rotation or circular dichroism of probe light. Chiral molecules are known to have differential absorption cross-sections for the 'left' and 'right' circularly polarized (LCP/RCP) light - known as circular dichroism. However, this differential absorption is typically of the order of $0.01 \%$ to the total absorption cross section and arises out of magnetic dipole and electric quadrupole interactions and interferences. Such experiments in gas phase are further plagued by low target densities and subsequently low signal/yields.

In 1976, Ritchie [1] reported that ionization of randomly oriented gas phases chiral molecules with circular polarized light results in an angular distribution of electrons that will show a (strong) linear term in $\cos (\theta)$ i.e. $I(\theta)=1+b_{1} \cos (\theta)+b_{2} \cos ^{2}(\theta)$ within the dipole approximation. Here, $\theta$ is the angle between the velocity of the ejected electron and the propagation direction of the circularly polarized light. This linear term in $\cos (\theta)$ manifests as excess in the scattering of electrons either in the forward or backward direction for a given enantiomer using left/right circularly polarized light. It has been extensively demonstrated in the last decade that photoelectrons from many chiral molecules upon one-photon ionization with CPL using synchrotron sources [2, 3] exhibit a strong chiral asymmetry (upto $32 \%$ [4]). This chiral asymmetry in the photoelectron angular distribution using

This is an Open Access article distributed under the terms of the Creative Commons Attribution License 2.0, which permits unrestricted use, distribution, and reproduction in any medium, provided the original work is properly cited. 
circularly polarized light is termed photo-electron circular dichroism (PECD). The high sensitivity of PECD makes angular-resolved photoionization of enantiomers very promising as a sensitive analytical technique to characterize chiral samples. While synchrotron light sources are large scale facilities, we have attempted to open the PECD technique to small scale laboratories with a table top setup using a femtosecond laser and position sensitive detectors. Here, we demonstrate that a relatively simple table-top photo-electron photo-ion coincidence imaging set-up [5-7] can be used to detect mass-selected chiral molecules with high selectivity and sensitivity with our results on the chiral molecule camphor.

\section{Experimental details}

Circular polarized light at $400 \mathrm{~nm}, 150 \mathrm{fs}$ long and a pulse energy of $30 \mu \mathrm{J}$ is used to photoionize the chiral molecules. The detection setup consists of a state of the art coincidence imaging setup for photoelectrons and photoions using the velocity map imaging technique. Both the electron and the coincident ion were detected on a single Micro-Channel-Plate delay-line detector, by switching the High-Voltages (HV) on the ion optics of the Velocity-Map-Ion (VMI) lenses [5-7]. Initial experiments were done on the two enantiomers of camphor that were obtained commercially (Sigma-Aldrich, purity $98 \%(\mathrm{R})$ and $99 \%(\mathrm{~S})$ ) and were contained in two separate tubes in vacuum lines outside the coincidence imaging setup. A set of gas valves made it possible to switch between an expansion with S-camphor or R-camphor. Experiments were performed by repeatedly switching between the two handedness of circular polarization.

\section{Results and Discussion}
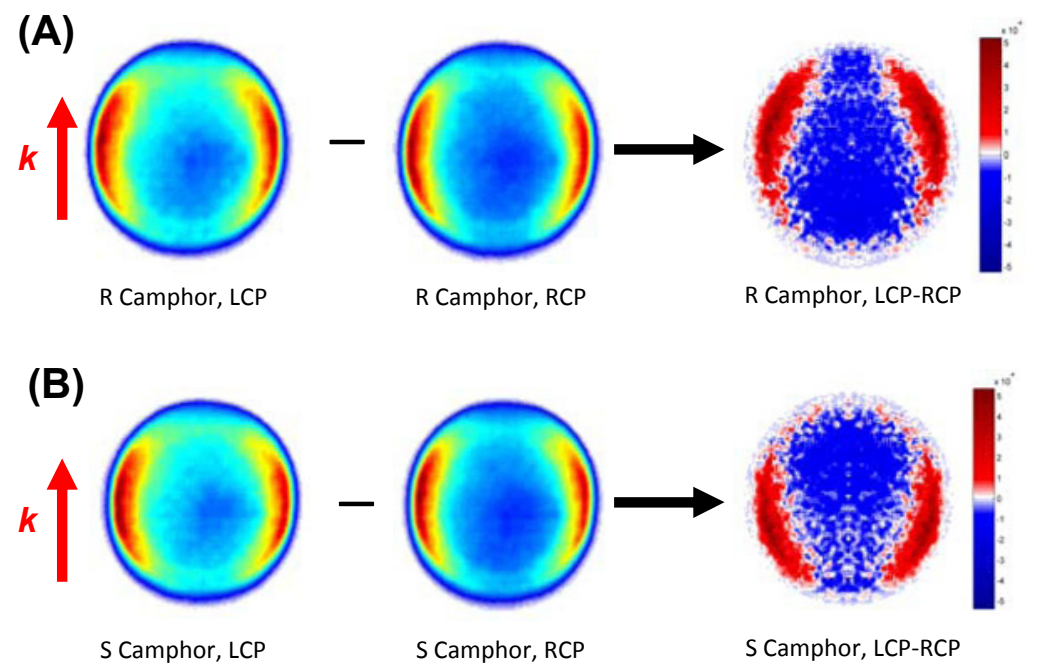

Fig. 1. Photoelectron images from (A) R-Camphor and (B) S-Camphor obtained from 3-photon ionization using LCP and RCP femtosecond pulses centered at $400 \mathrm{~nm}$. Difference of images of LCP and RCP for each enantiomer $(\mathrm{R}$ or $\mathrm{S})$ shows the forward backward asymmetry $(\sim 8 \%)$ arising due to chiral molecular potential of camphor. The red arrow shows the laser propagation direction, which is the symmetry axis in experiments with circular polarized light.

Photo excitation of Camphor molecules ( $\mathrm{R}$ and $\mathrm{S}$ enantiomers; Ionization Potential $=8.8 \mathrm{eV}$ ) at $400 \mathrm{~nm}$ leads to 3-photon ionization producing photoelectrons of close to $0.5 \mathrm{eV}$. We imaged the 
photoelectrons from R and S enantiomers with both light helicities - LCP and RCP, see Figure 1 (A) and (B). The difference of images of LCP and RCP for a given enantiomer shows a very strong angular asymmetry of photoelectrons corresponding to ionization of the camphor HOMO orbital. To obtain a quantitative measure of the forward backward asymmetry, we integrate all photoelectron events in the forward and backward hemispheres in both images (i.e. $I_{\text {lcp,forw }}, I_{\text {lcp,back, }}, I_{r c p \text {,forw }}$ and Ilcp,back $)$ and determine the asymmetry in events by evaluating $\left(\mathrm{I}_{\mathrm{lcp}, \text { forw }}-\mathrm{I}_{\mathrm{rcp}, \text { forw }}\right)-\left(\mathrm{I}_{\mathrm{lcp}, \text { back }}-\mathrm{I}_{\mathrm{rcp}, \text { back }}\right)$. We relate this difference in the integrated intensities in the forward/ backward hemisphere to the total integrated intensity of observed photoelectrons. This definition of the overall multi-photon PECD effect is consistent with the one-photon PECD definition [3]. Our data for Camphor at $400 \mathrm{~nm}$ shows the multi-photon PECD $=7.8 \%$. This is a factor of 3 larger than the effect measured in onephoton ionization using synchrotron light sources at the same photoelectron kinetic energy [3]. Our experimental results are consistent with recent findings of Lux and co-workers from the Kassel group, Germany [8].

Our data on photoelectron forward-backward chiral asymmetry from gas phase camphor molecules shows a significant asymmetry of about $8 \%$ obtained with a table top femtosecond laser source and a coincidence imaging setup. This novel technique of multiphoton PECD can be easily extended to probe for effects of the magnitude of photoelectron energy by changing the wavelength of the femtosecond excitation laser pulse using non-linear frequency mixing techniques. It will be very interesting to investigate whether there is a change in the size of the MPMS-PECD effect by varying the kinetic energy of the ejected photoelectron. Such effects have been observed in PECD measurements using synchrotron radiation [3], and we are presently investigating these photoelectron energy effects in our novel microscope. Furthermore, the availability of a multi-photon excitation scheme also offers the possibility to explore the influence of (near)-resonance excitation at intermediate excited levels and alignment of the intermediate state. By going via an electronically excited state one can probe the effect of the initial molecular orbital on the magnitude of the chiral asymmetry in order to maximize the MPMS-PECD effect. This can be readily explored by varying the femtosecond laser wavelength, or by using a two-color femtosecond pump-probe excitation. The technique can be combined with femtosecond (polarization) pulse shaping and such further extensions using pulse shaping are currently planned in our laboratory. More experiments and analysis are in progress.

\section{References}

1. B. Ritchie, Phys. Rev. A 13, 1411 (1976).

2. I. Powis, J. Phys. Chem. A 104, 878 (2000).

3. L. Nahon, G. Garcia, H. Soldi.-Lose, S. Daly, and I. Powis, Phys. Rev. A 82, 032514 (2010).

4. S. Daly, I. Powis, G. Garcia, H. Soldi-Lose and L. Nahon, J. Chem. Phys. 134, 064306 (2011)

5. A. Vredenborg, W. G. Roeterdink, and M. H. M. Janssen, Rev. Sci. Instrum. 79, 063108 (2008).

6. A. Vredenborg, C. S. Lehmann, D. Irimia, W. G. Roeterdink, M. H. M. Janssen,

ChemPhysChem., 12, 1459 (2011).

7. C. S. Lehmann, N. Bhargava Ram, D. Irimia and M. H. M. Janssen, Faraday Discuss., 153, 173 (2011).

8. C. Lux, M. Wollenhaupt, T. Bolze, Q. Liang, J. Koehler, C. Sarpe and T. Baumert, Angew. Chem. Int. Ed., 51, 4755 (2012) 